\title{
Knockdown of KIF26B inhibits breast cancer cell proliferation, migration, and invasion
}

This article was published in the following Dove Press journal:

OncoTargets and Therapy

\section{Shudong $\mathrm{Gu}^{1, *}$ \\ Haibin Liang ${ }^{2, *}$ \\ Donghui $\mathrm{Qi}^{3}$ \\ Liyan $\mathrm{Mao}^{4}$ \\ Guoxin Mao' \\ Li Qian' \\ Shu Zhang ${ }^{5}$}

'Department of Oncology, Affiliated Hospital of Nantong University, Nantong 22600I, China; ${ }^{2}$ Department of General Surgery, Xinhua Hospital, Shanghai Jiao Tong University School of Medicine, Shanghai 200092,

China; ${ }^{3}$ Medical College of Nantong University, Nantong 22600I, China; ${ }^{4}$ Department of Endoscopic Diagnosis and Treatment of Digestive Diseases, Xinhua Hospital, Shanghai Jiao Tong University School of Medicine, Shanghai 200092, China; ${ }^{5}$ Department of Pathology, Affiliated Hospital of Nantong University, Nantong 22600I, China

*These authors contributed equally to this work

Correspondence: Li Qian Department of Oncology, Affiliated Hospital of Nantong University, 20 Xisi Road, Nantong 22600I, Jiangsu Province, China

Email qianlint@foxmail.com

Shu Zhang

Department of Pathology, Affiliated Hospital of Nantong University, 20 Xisi Road, Nantong 22600I, Jiangsu Province, China Email YLTD66@। 26.com
Background: Kinesin family member 26B (KIF26B) plays a key role in the development and progression of many human cancers. However, the role and underlying mechanisms of KIF26B in breast cancer cells remain unknown.

Materials and methods: In this study, we inhibited the expression of KIF26B in MDA-MB231 and MCF-7 cells using lentivirus-delivered shRNA.

Results: Lentivirus-mediated KIF26B knockdown significantly suppressed cell proliferation, colony formation, migration, and invasion. Furthermore, cell cycle analyses revealed that the percentage of cells in the G0/G1 phase was significantly increased in KIF26B knockdown cells. Moreover, the knockdown of KIF26B significantly promoted cell apoptosis via the upregulation of cleaved caspase- 3 and Bax.

Conclusion: Our data indicate that KIF26B plays a pivotal role in tumor growth and metastasis in breast cancer cells and may be a potential therapeutic target for treating breast cancer.

Keywords: KIF26B, breast cancer cell, proliferation, migration, invasion

\section{Introduction}

Breast cancer is one of the most frequent malignant tumors that occurs in women; worldwide, there are more than 1.7 million new cases each year. ${ }^{1-3}$ Although improvements in basic and clinical research have decreased the mortality rates of breast cancer in recent years, its prognosis remains poor. ${ }^{4}$ This may be attributed to its incomplete diagnosis, frequent recurrence, and high chemoresistance, as well as the adverse effects of surgeries. ${ }^{5-7}$ Therefore, it is important to identify novel therapeutic targets and factors that could be potential biomarkers for the early diagnosis and prognosis of patients with breast cancer.

The kinesin superfamily proteins (KIFs) are a conserved class of molecular motor proteins with microtubule binding and ATPase activities. ${ }^{8}$ The kinesin family member 26B (KIF26B) gene belongs to the N-type kinesin family and encodes a 21,088amino acid protein. ${ }^{9}$ An increasing amount of evidence demonstrates the importance of KIF26B in the regulation of many physiological events, including brain function, developmental patterning, and tumorigenesis. Uchiyama et $\mathrm{al}^{10}$ reported that KIF26B is essential for kidney development because it regulates the adhesion and polarization of mesenchymal cells; moreover, the transcription of KIF26B is regulated by Sall1. In the neuronal system, KIF26B transports Abelson interactor protein 1 (Abi-1) to different cellular compartments, particularly to the postsynaptic density of excitatory synapses. ${ }^{11}$ Recently, a number of studies have reported that the abnormal expression of KIF26B plays a key role in the development or progression of a number of human cancers, including esophageal adenocarcinoma, ${ }^{12}$ colorectal cancer, ${ }^{13}$ 
and gastric cancer. ${ }^{14}$ Wang et $\mathrm{al}^{15}$ demonstrated that KIF26B was overexpressed in breast cancer tissues and that breast cancer patients with high KIF26B expression had a shorter survival time. However, the biological role of KIF26B and its mechanism of regulation in breast cancer have not been investigated. Thus, whether KIF26B regulates the proliferation or metastatic ability of breast cancer cells and its potential mechanism are unknown.

In the current study, we investigated the effects of KIF26B knockdown on breast cancer cell proliferation, migration, and invasion, as well as breast cancer cell apoptosis and cell cycle distribution. We also investigated the potential mechanism of KIF26B.

\section{Materials and methods}

\section{Cell lines and cell culture} MDA-MB-231, SK-BR-3, BT-474, MCF-10A, and MCF-7 cells were purchased from the Chinese Academy of Science Cell Bank (Shanghai, China). The cells were cultured in high-glucose DMEM containing 10\% fetal bovine serum (Thermo Fisher Scientific, Waltham, MA, USA) and supplemented with antibiotics $(100 \mathrm{U} / \mathrm{mL}$ penicillin and $100 \mathrm{mg} / \mathrm{mL}$ streptomycin; Sigma-Aldrich Co., St Louis, MO, USA) at $37^{\circ} \mathrm{C}$ and $5 \% \mathrm{CO}_{2}$ in a humidified incubator.

\section{RNA isolation and quantitative reverse transcription polymerase chain reaction assays}

Total cellular RNA was extracted using TRIzol reagent (Thermo Fisher Scientific) according to the manufacturer's protocol. First-strand cDNA was synthesized using the PrimeScript RT reagent kit (Takara, Shiga, Japan). Quantitative reverse transcription polymerase chain reaction (qRT-PCR) was performed using SYBR Green Premix Ex Taq (Takara) according to the manufacturer's protocol. The relative expression levels of genes were calculated using the $\Delta \Delta \mathrm{Ct}$ method, and GAPDH was used as the endogenous reference gene. The following primer sequences were used: KIF26B forward, 5'-GCTGCGTGTTCTGTTTCGG-3' and reverse, 5'-TTCCTTGCGTTCGTTTATGAG-3'; Bax forward, 5'-CCCGAGAGGTCTTTTTCCGAG-3' and reverse, 5'-CCAGCCCATGATGGTTCTGAT-3'; Bcl-2 forward, 5'-GGTGGGGTCATGTGTGTGG-3' and reverse, 5'-CGGTTCAGGTACTCAGTCATCC-3'; cyclin D1 forward, 5'-TGGAGCCCGTGAAAAAGAGC-3' and reverse, 5'-TCTCCTTCATCTTAGAGGCCAC-3'; and GAPDH forward, 5'-AGAAGGCTGGGGCTCATTTG-3' and reverse,
5'-AGGGGCCATCCACAGTCTTC-3'. Three independent experiments were performed.

\section{Colony formation assays}

MDA-MB-231 and MCF-7 cells were infected for $72 \mathrm{~h}$ and seeded in 6-well plates at a density of 500 cells/well. After culture for 14 days, the cells were fixed with $4 \%$ paraformaldehyde for $20 \mathrm{~min}$ and stained with $0.1 \%$ crystal violet (Beyotime, Shanghai, China) for $25 \mathrm{~min}$. After the cells were washed with PBS and air dried, the colonies were manually counted and photographed under a microscope.

\section{In vitro Transwell cell migration/invasion assay}

Migration and invasion assays were performed as described by Xie and Wang. ${ }^{16}$ Briefly, $4 \times 10^{4}$ and $1 \times 10^{5}$ lentivirustransfected MDA-MB-231 and MCF-7 cells, respectively, were seeded in the upper chambers of $8-\mu \mathrm{m}$ pore size inserts with or without Matrigel (Corning Incorporated, Corning, NY, USA) in a 24-well plate. Then, the cells were fixed in $4 \%$ paraformaldehyde and stained with $0.1 \%$ crystal violet. Cells that had penetrated the membranes were quantified in five random microscopic fields at $100 \times$ magnification under a microscope. Three independent experiments were performed.

\section{Cell apoptosis assay}

Apoptosis was performed using an annexin V/propidium iodide (PI) apoptosis kit according to the manufacturer's protocol (Thermo Fisher Scientific). Briefly, MDA-MB-231 and MCF-7 cells were infected with KIF26B shRNA or negative control RNA. Then, the cells were placed in 6-well culture plates $\left(5 \times 10^{4}\right.$ cells $\left./ \mathrm{mL}\right)$. After $48 \mathrm{~h}$, the cells were harvested by trypsinization without EDTA, washed with cold PBS, and resuspended in $100 \mu \mathrm{L}$ of binding buffer at a density of $1 \times 10^{6}$ cells $/ \mathrm{mL}$. Then, $5 \mu \mathrm{L}$ of annexin $\mathrm{V}$-fluorescein isothiocyanate (FITC) and $5 \mu \mathrm{L}$ of a PI working solution $(100 \mu \mathrm{g} / \mathrm{mL})$ were added to the cells, gently mixed, and then incubated in the dark for $15 \mathrm{~min}$ at room temperature. Afterward, $400 \mu \mathrm{L}$ of binding buffer was added, and the cells were immediately analyzed by flow cytometry. This experiment was repeated three times.

\section{Cell cycle analysis}

Breast cancer cells were seeded in $60-\mathrm{mm}$ culture plates. After transfection with KIF26B shRNA or control RNA for $48 \mathrm{~h}$, the cells were harvested by trypsinization, washed twice with cold PBS, and fixed in $70 \%$ ethanol at $4^{\circ} \mathrm{C}$ overnight. After fixation, the cells were rinsed with PBS and incubated 
with $10 \mathrm{mg} / \mathrm{mL}$ RNase and $1 \mathrm{mg} / \mathrm{mL}$ PI (Sigma-Aldrich Co.) at $37^{\circ} \mathrm{C}$ for $30 \mathrm{~min}$ in the dark. The cell cycle distribution was measured and analyzed using Cell Quest acquisition software (BD Biosciences, San Jose, CA, USA). Each experiment was performed in triplicate.

\section{Lentivirus-mediated RNA interference}

The siRNA sequence targeted to KIF26B was 5'-CGGAC AGCCTCTCCTATTA-3', as reported previously, ${ }^{15}$ and the negative control sequence was $5^{\prime}$-TTCTCCG AACGTGTCACGT-3'. Recombinant lentiviruses expressing KIF26B shRNA or negative control shRNA were provided by GeneChem (Shanghai, China). Cells were infected with lentivirus for $72 \mathrm{~h}$ and then selected with puromycin for 7 days. The expression level of KIF26B was analyzed by qRT-PCR and western blotting.

\section{Western blot analysis}

Total protein was isolated from the cultured cell lines using radioimmunoprecipitation assay lysis buffer (Beyotime Biotechnology, Shanghai, China). The protein concentration in the cell extracts was measured using a bicinchoninic acid protein assay kit (Beyotime Biotechnology) with bovine serum albumin as a standard. Equal amounts of protein $(40 \mu \mathrm{g})$ were separated by sodium dodecyl sulfate polyacrylamide gel electrophoresis and then transferred onto polyvinylidene difluoride (PVDF) membranes (EMD Millipore, Billerica, MA, USA). The membranes were blocked in 5\% fat-free milk and then incubated with the following primary antibodies at $4{ }^{\circ} \mathrm{C}$ overnight: anti-KIF26B (Abcam, Cambridge, UK), anti-GAPDH (Abways, Shanghai, China), anti-Bcl-2 (Cell Signaling Technology, Danvers, MA, USA), anti-Bax (Cell Signaling Technology), anti-cyclin D1 (Cell Signaling Technology), anti-p-Rb (ser780) (Cell Signaling Technology), anti-cleaved caspase-3 (Cell Signaling Technology), anti-E-cadherin (Cell Signaling Technology), anti-N-cadherin (Cell Signaling Technology), anti-Vimentin (Cell Signaling Technology), and anti-Snail (Cell Signaling Technology); then, the membranes were incubated with the secondary detection antibody (Cell Signaling Technology) for $1 \mathrm{~h}$ at room temperature. Protein bands were visualized using an enhanced chemilumescent (ECL) reagent (Pierce Biotechnology, Waltham, MA, USA).

\section{Statistical analysis}

All statistical analyses were carried out using SPSS 19.0 software (IBM Corporation, Armonk, NY, USA). The statistical significance of the qRT-PCR results for KIF26B gene expression and the results for cell proliferation, colony formation, cell cycle, migration, invasion, and apoptosis were calculated using Student's $t$-tests (two-tailed). All values are expressed as the mean $\pm \mathrm{SD}$ unless otherwise stated. A $p$-value $<0.05$ was considered to be statistically significant.

\section{Results}

\section{Inhibition of KIF26B in the MCF-7 and MDA-MB-23 I cell lines}

To investigate the expression of KIF26B in a large cohort of patient samples, we retrieved and analyzed the expression of KIF26B in The Cancer Genome Atlas database from breast cancer using the online web portal UALCAN (http:// ualcan.path.uab.edu). As shown in Figure 1A, KIF26B was significantly upregulated in luminal, HER2-positive, and triple-negative breast cancer tissues compared with normal tissues. In addition, KIF26B showed highest expression in luminal breast tumors. In order to examine the functional role of KIF26B in breast cancer cells, we performed qRT-PCR analysis of KIF26B in 4 breast cancer cell lines and the normal breast cancer cell line MCF-10A. As shown in Figure 1B, the mRNA level of KIF26B in these cell lines was differentially expressed, with its high expression levels in MDA-MB-231 and MCF-7 cells and lowest level in MCF$10 \mathrm{~A}$ cells. To examine the underlying role of KIF26B in tumorigenesis in breast cancer, lentiviral RNA interference (shKIF26B) was used to suppress KIF26B in MCF-7 and MDA-MB-231 cells; an empty lentiviral vector was used as a negative control (shNC). The transfection efficiency was approximately $92 \%$ in MCF-7 cells and 95\% in MDA-MB231 cells (Figure 1C). Furthermore, both the mRNA and protein levels of KIF26B were significantly downregulated in shKIF26B compared to the shNC group or the control group in the MCF-7 and MDA-MB-231 cell lines (Figure 1D and $\mathrm{E}, p<0.01)$.

\section{Knockdown of KIF26B decreases the proliferation and colony formation capacities of breast cancer cells}

To identify the potential effect of KIF26B on cell proliferation, we performed CCK8 assays in MCF-7 and MDAMB-231 cells. The results showed that KIF26B knockdown MCF-7 and MDA-MB-231 cells had a significantly lower proliferation rate than that of control cells ( $p<0.01, p<0.05$, Figure 2A). Furthermore, colony formation assays revealed that smaller and fewer colonies were observed in the shKIF26B group compared with the shNC group $(p<0.01$, Figure 2B and C). These data indicate that KIF26B might play a crucial role in breast cancer cell proliferation. 

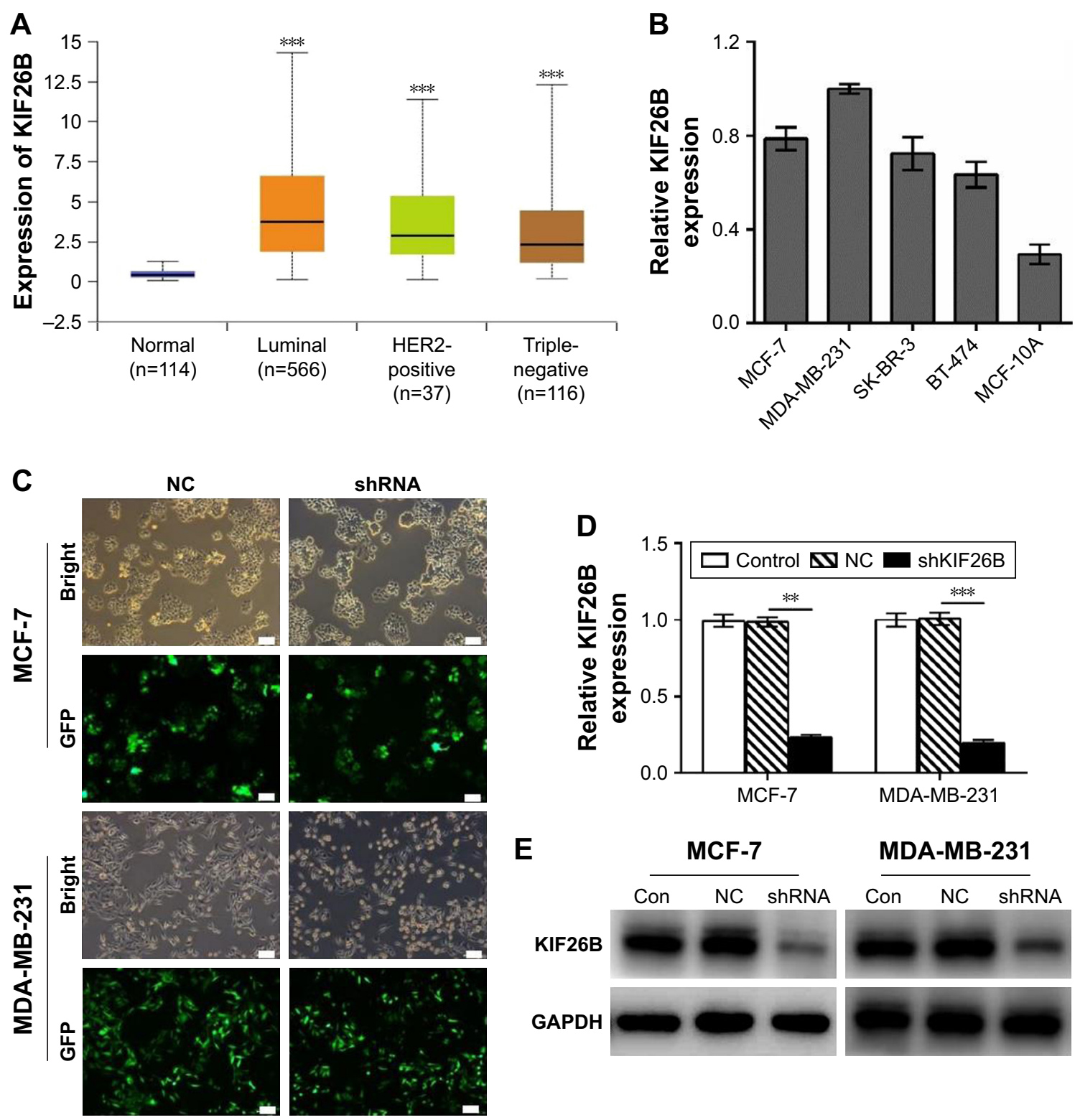

$\mathbf{E}$

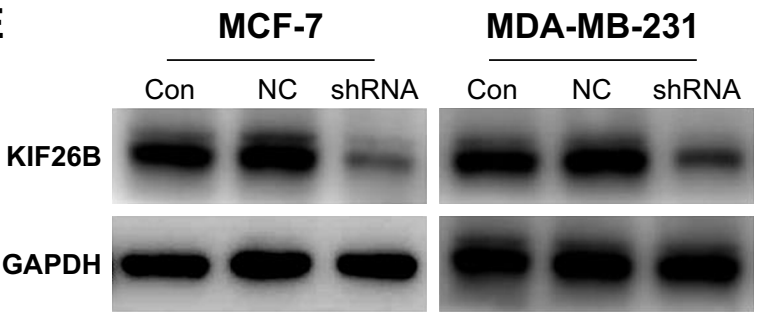

Figure I Lentivirus-mediated gene knockdown of KIF26B in breast cancer cells.

Notes: (A) Boxplot showing relative expression of KIF26B in normal, luminal, HER2-positive, and triple-negative breast cancer patients. *** $p<0.001$. (B) qRT-PCR analysis of KIF26B in 4 breast cancer cell lines and the normal breast cell line MCF-IOA. GAPDH served as the internal control. (C) Microscopic images of MCF-7 and MDA-MB-23I cells infected with control lentivirus or shKIF26B lentivirus (scale bar, $100 \mu \mathrm{m}$ ). (D) qRT-PCR analysis of KIF26B mRNA expression in the indicated cells; GAPDH served as the internal control. ${ }^{* *} p<0.01$ and $* * * p<0.00 I$. (E) The knockdown efficiency of KIF26B shRNA in MCF-7 and MDA-MB-23I cells was confirmed by western blot analysis. GAPDH served as the loading control.

Abbreviations: Con, control; HER2, human epidermal growth factor receptor 2; KIF26B, kinesin family member 26B; NC, negative control; qRT-PCR, quantitative reverse transcription polymerase chain reaction; GFP, green fluorescent protein.

\section{Knockdown of KIF26B induces breast cancer cell apoptosis}

Next, we examined the effects of KIF26B knockdown on cell apoptosis by flow cytometry (Figure 3A). As shown in Figure 3B, KIF26B knockdown significantly reduced the number of surviving cells and increased the number of apoptotic cells compared with those in the shNC group. To further investigate the underlying molecular mechanism of shKIF26B-induced apoptosis in MCF-7 and
MDA-MB-231 cells, we measured the expression of apoptosisrelated proteins after KIF26B knockdown. As shown in Figure 3C, lentivirus-mediated knockdown of KIF26B led to the upregulation of Bax and cleaved caspase- 3 expression and the downregulation of Bcl-2 expression, which agreed with our previous flow cytometry results. Consistent with the western blot results, knockdown of KIF26B results in the increased mRNA levels of Bax and reduced mRNA levels of Bcl-2 (Figure 3D). The results suggest that the knockdown 
A

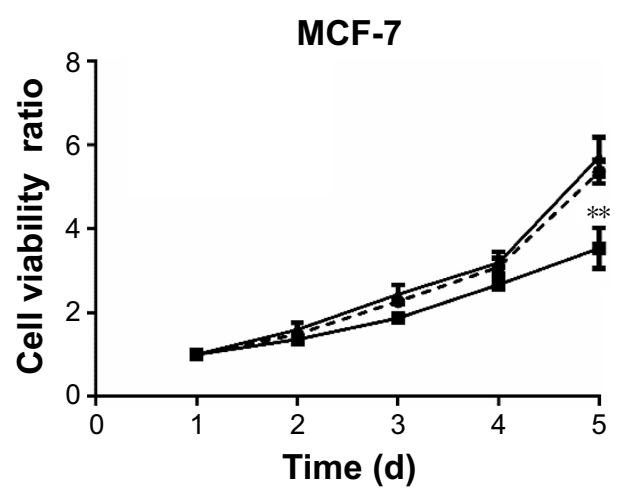

MDA-MB-231

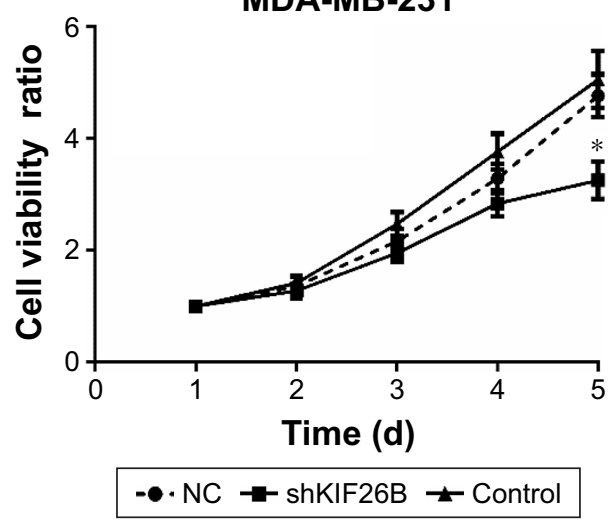

B

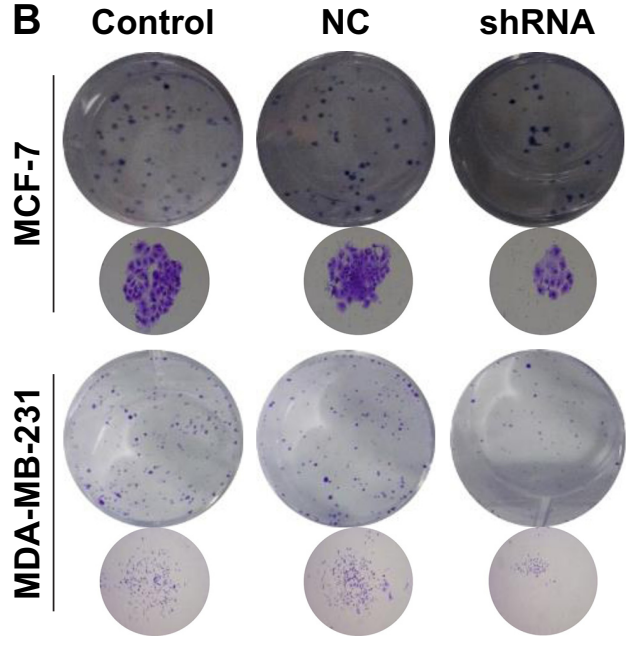

C

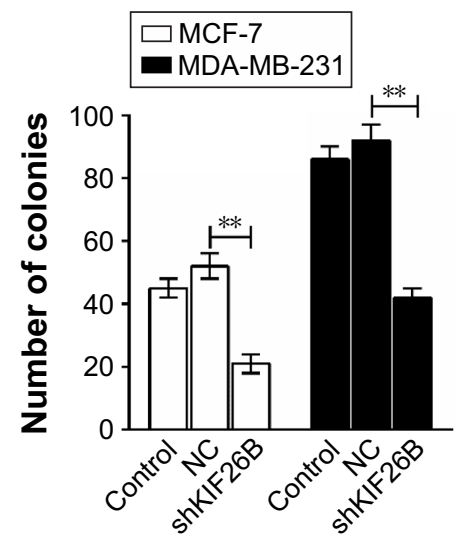

Figure 2 Depletion of KIF26B suppressed the proliferation and colony formation abilities of breast cancer cells.

Notes: (A) Cell viability was measured by CCK8 assay in MCF-7 and MDA-MB-23I cells after KIF26B knockdown. (B) Knockdown of KIF26B decreased the colony formation activities of MCF-7 and MDA-MB-23I cells. Cell colonies were stained with crystal violet and photographed. (C) The number of colonies was counted and statistically analyzed. $* p<0.05$, ** $p<0.01$.

Abbreviations: CCK8, cell counting kit-8; d, days; KIF26B, kinesin family member 26B; NC, negative control.

of KIF26B promotes cell apoptosis by regulating apoptosisrelated proteins.

\section{Knockdown of KIF26B arrests the cell cycle in the G0/GI phase}

To examine the reason for the shKIF26B-mediated inhibition of cell proliferation, we analyzed the cell cycle by flow cytometry. Compared to the shNC group, the percentage of cells in the G0/G1 phase was significantly increased in shKIF26B-transfected MCF-7 and MDA-MB-231 cells (Figure 4A and B), suggesting that KIF26B knockdown induced G0/G1 phase arrest. In addition, we observed a dramatic decrease in the protein expression of cyclin D1 and $\mathrm{p}-\mathrm{Rb}$ in MCF-7 and MDA-MB-231 cells after the downregulation of KIF26B (Figure 4C), which is consistent with the G0/G1 phase arrest induced by shKIF26B. Consistently, the mRNA level of cyclin D1 showed a significant decrease after knockdown of KIF26B (Figure 4D). Taken together, these data suggest that $\mathrm{G} 0 / \mathrm{G} 1$ phase arrest might contribute to the suppression of cell proliferation induced by the knockdown of KIF26B.

\section{Knockdown of KIF26B inhibits tumor metastasis and invasion by modulating EMT-related genes}

To investigate the effect of KIF26B on the migration and invasion of breast cancer cells, we conducted Transwell migration and Matrigel invasion assays. As shown in Figure $5 \mathrm{~A}$ and $\mathrm{B}$, cell migration and the invasive capabilities of KIF26B knockdown cells were significantly decreased compared with those of control cells. To explore the potential mechanisms underlying the shKIF26B-induced inhibition of migration and invasion, the expression of EMT-related proteins was detected by western blot. We found that KIF26B knockdown increased the expression of E-cadherin but decreased the expression of $\mathrm{N}$-cadherin, vimentin, 
A

MCF-7

MDA-MB-231
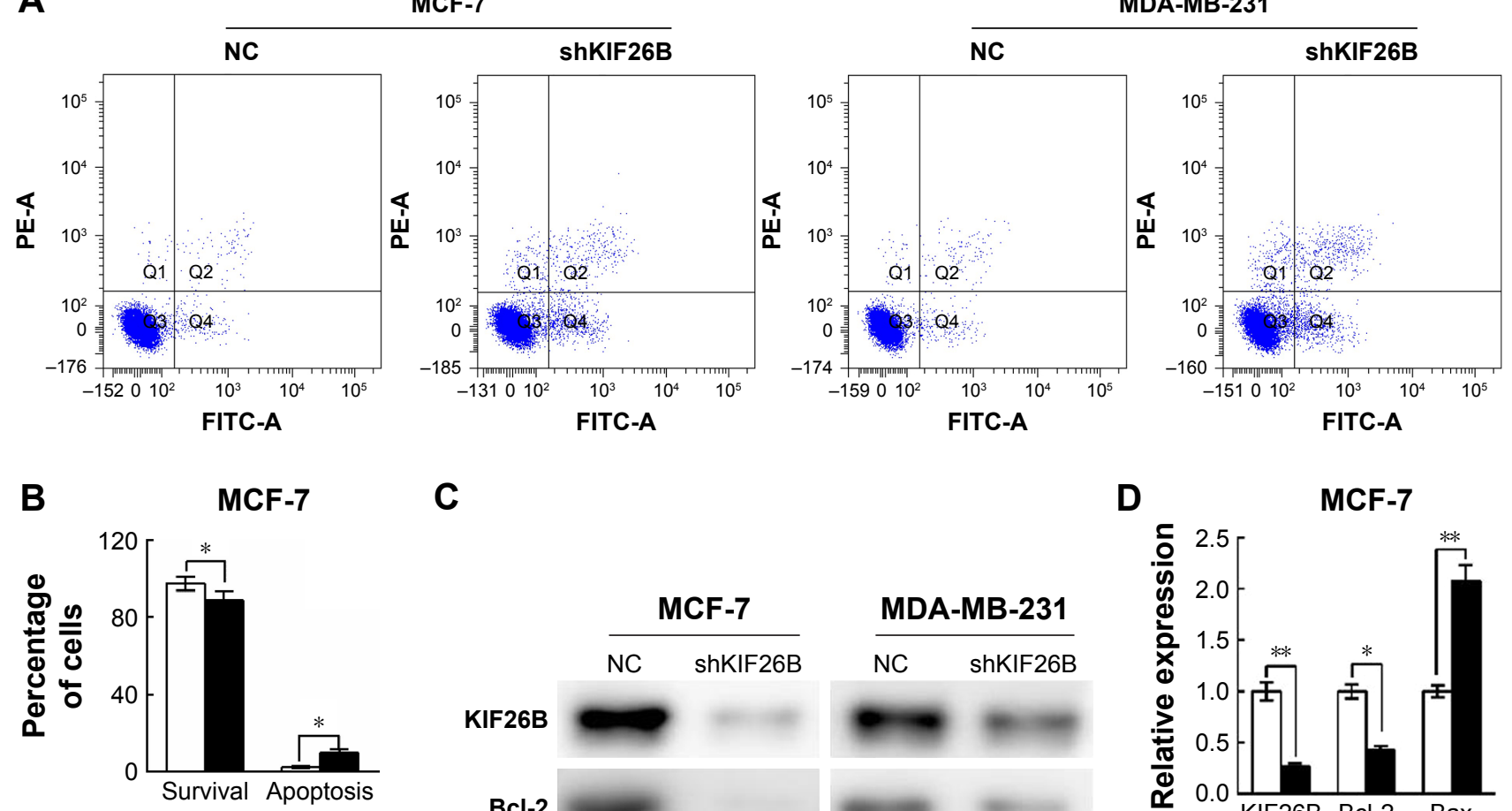

C

D
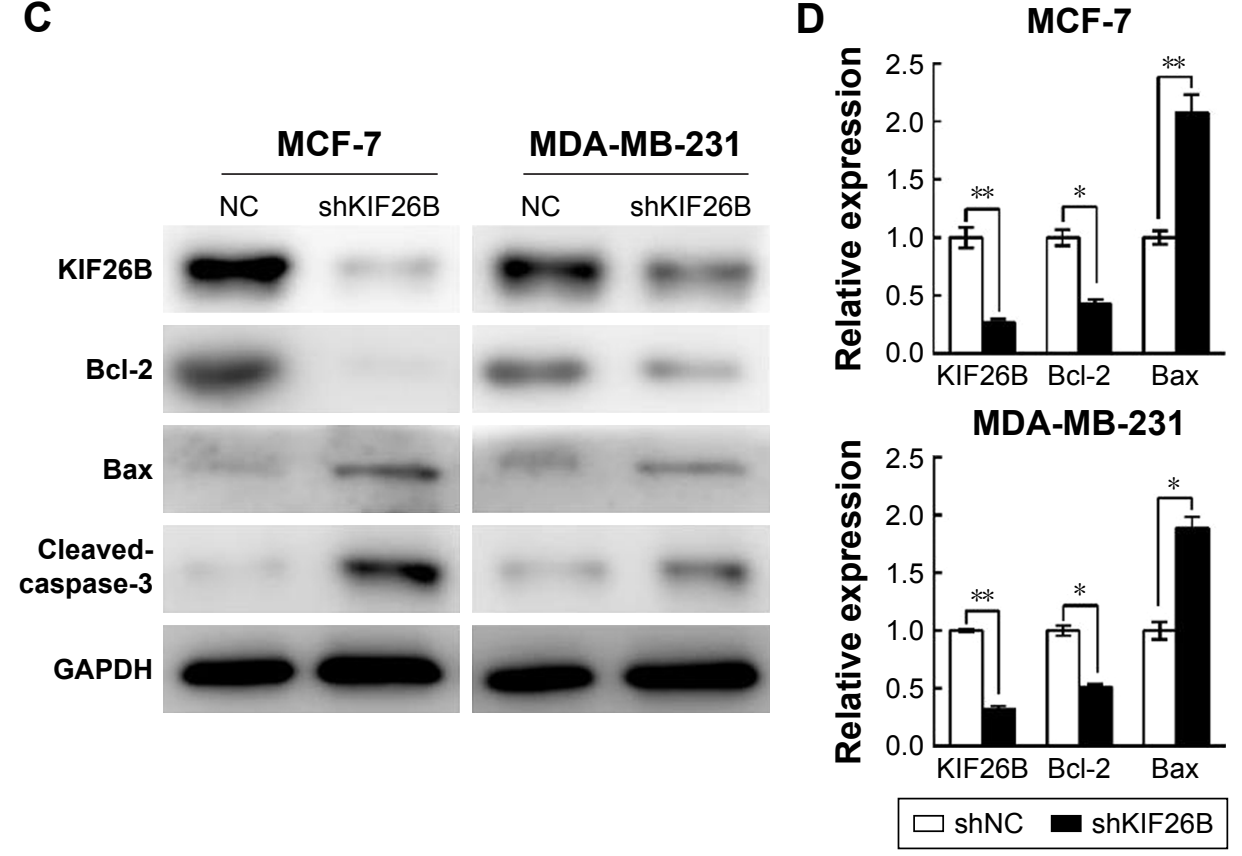

Figure 3 Downregulation of KIF26B induced cell apoptosis in breast cancer cells.

Notes: (A) Flow cytometry analysis of breast cancer cell apoptosis using annexin V-FITC and PI dual labeling with or without KIF26B knockdown. (B) The percentage of apoptotic cells was calculated. The data are shown as the mean \pm SD. (C) The expression levels of KIF26B, Bcl-2, Bax, and cleaved caspase-3 in MCF-7 cells (left panel) and MDA-MB-23I cells (right panel) were measured by western blotting. (D) qRT-PCR analysis of KIF26B, Bcl-2, and Bax expression in MCF-7 and MDA-MB-23I cells. Each experiment was repeated three times. ${ }^{*} p<0.05$ and $* * p<0.01$ compared with the negative control group.

Abbreviations: FITC, fluorescein isothiocyanate; KIF26B, kinesin family member 26B; NC, negative control; PI, propidium iodide; qRT-PCR, quantitative reverse transcription polymerase chain reaction; FITC-A, fluorescein isothiocyanate-Annexin V; PE-A, Phycoerythrin-area.

and Snail1 (Figure 5C). Thus, the above results suggest that KIF26B plays an important role in breast cancer cell migration and invasion.

\section{Discussion}

The KIFs play important roles in proliferation and migration of breast cancer cells. Previous reports demonstrated that elevated expression of KIF2A and KIF14 was positively associated with lymph nodes of patients with breast cancer. ${ }^{17,18}$ Knockdown of KIF3C in breast cancer cell lines inhibited epithelial-mesenchymal transition (EMT) and metastasis. ${ }^{19}$ In addition, silencing of $\mathrm{KIF} 3 \mathrm{C}$ reduced proliferation of breast cancer cells via inducing G2/M phase arrest. ${ }^{19}$ It has been reported that KIF26B plays an important role in many human cancers. ${ }^{12-14}$ KIF26B is highly expressed in breast cancer tissues, and high KIF26B expression is correlated with poor prognosis. ${ }^{15}$ This evidence suggests that KIF26B might play an instrumental role in tumorigenesis in breast cancer cells. In this study, for the first time, we examined the oncogenic function of KIF26B and its underlying mechanism in breast cancer cells.

To better understand the underlying mechanism of shKIF26B-induced cell growth inhibition, we performed cell cycle distribution experiments after KIF26B knockdown and found that the knockdown of KIF26B induced G0/G1 
A

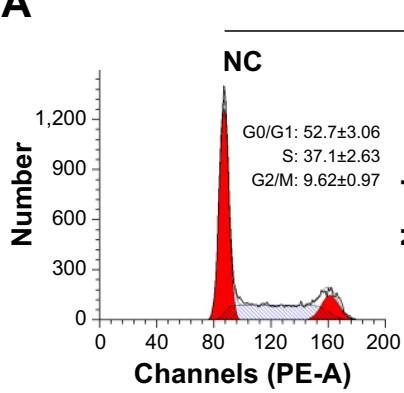

MCF-7

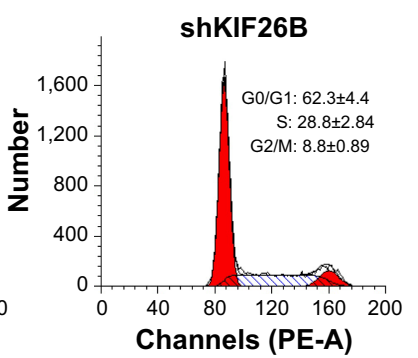

MDA-MB-231

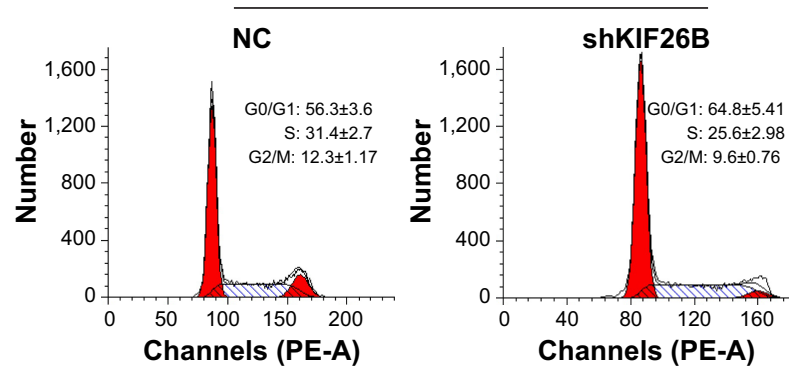

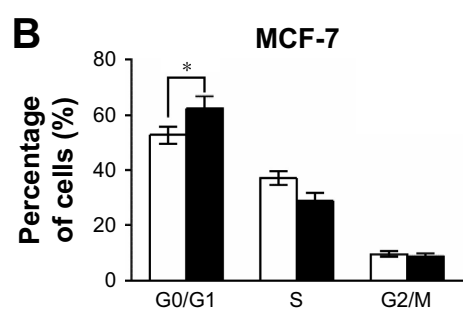

$\square$ Dip G1 $\square$ Dip G2 $\square$ Dip S

C
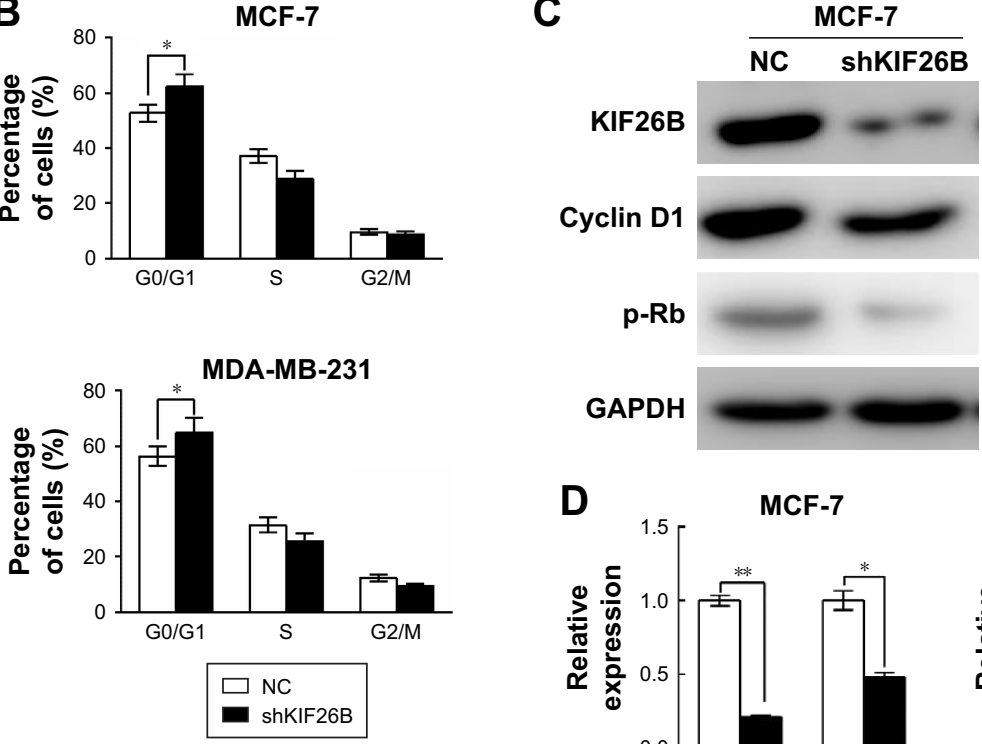

GAPDH

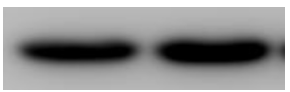

D
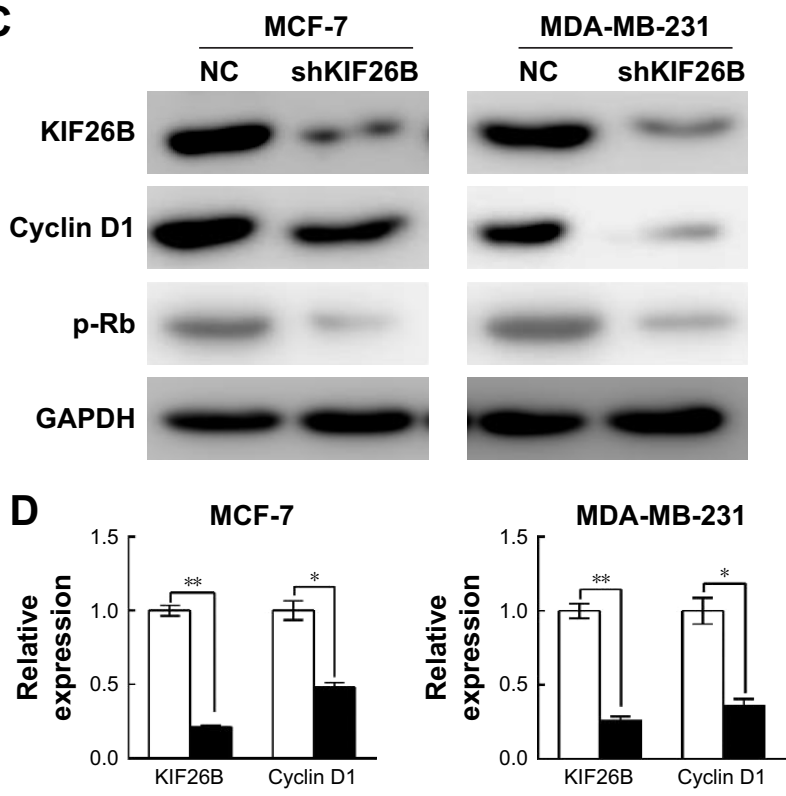

$\square$ shNC $\square$ shKIF26B

Figure 4 Knockdown of KIF26B arrested the cell cycle at the G0/GI phase.

Notes: (A) Flow cytometry analysis of the cell cycle progression in MCF-7 and MDA-MB-23I cells infected with control virus (NC) or shKIF26B virus. (B) The populations of cells in the G0/GI, S, and G2/M phases were calculated. The results are presented as the mean \pm SD. * $p<0.05$ compared with the negative control group. (C) Protein expression levels of cyclin DI and p-Rb were decreased in MCF-7 cells (left panel) and MDA-MB-23I cells (right panel) after KIF26B knockdown. (D) mRNA levels of KIF26B and cyclin DI were determined by qRT-PCR. ** $p<0.01$.

Abbreviations: KIF26B, kinesin family member 26B; NC, negative control; qRT-PCR, quantitative reverse transcription polymerase chain reaction; PE-A, Phycoerythrinarea; Dip, diploid.

phase arrest. It is well known that the activation of cell cycle phases is dependent on the regulation of cyclins and cyclindependent kinases (CDKs). ${ }^{20,21}$ The complex of cyclin D1 and CDK4 promotes the transition of cells from the G0/G1 phase to the $\mathrm{S}$ phase by phosphorylating the retinoblastoma tumor suppressor gene. ${ }^{22}$ Our results demonstrated that the knockdown of KIF26B resulted in a decrease in the expression of cyclin D1, which might be the reason for $\mathrm{G} 0 / \mathrm{G} 1$ arrest.

Apoptosis is considered to be one of the main contributors to cancer development. In apoptosis progression, caspase-3 is a crucial executioner caspase that is activated by the cleavage of caspase- 8 or caspase- $9 .{ }^{23}$ Activated caspase- 3 cleaves several downstream effectors, including the PARP protein, which eventually induces apoptosis. In the present study, cleaved caspase-3 was upregulated in the KIF26B knockdown group. Moreover, the proapoptotic Bax was increased, whereas the antiapoptotic Bcl-2 was decreased in KIF26B knockdown cells. These data suggest that the mitochondrial pathway was involved in shKIF26B-induced apoptosis.

EMT is a multistep plasticity process that plays a crucial role in cancer metastasis. EMT involves the loss of epithelial properties, such as decreased E-cadherin expression, and the gain of mesenchymal properties, such as increased $\mathrm{N}$-cadherin and vimentin expression. ${ }^{24,25}$ In this study, we found that the knockdown of KIF26B inhibited cell metastasis by increasing the expression of the epithelial marker E-cadherin and by decreasing the expression of the mesenchymal markers vimentin, Snail1, and N-cadherin. 
A

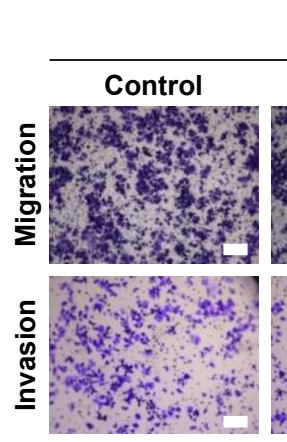

B
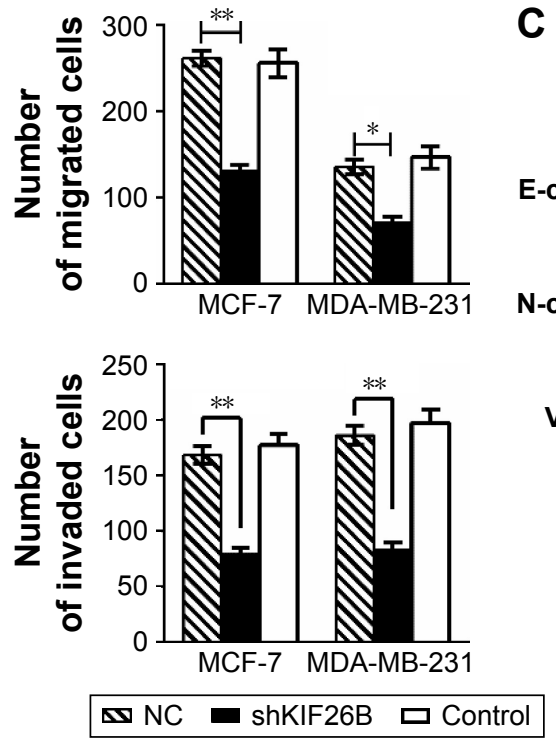

C
MCF-7
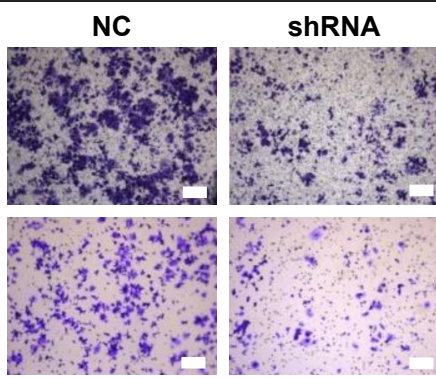

MDA-MB-231
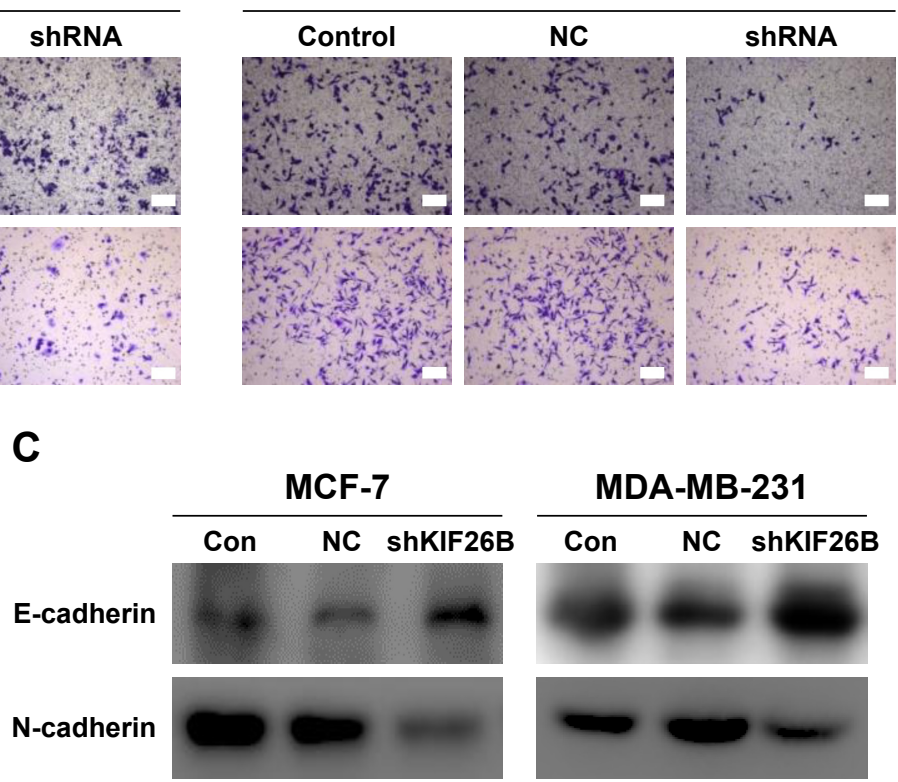

Vimentin

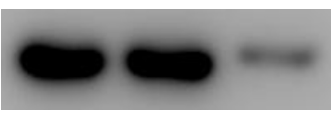

Snail1

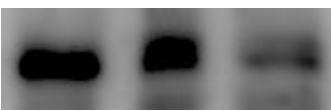

GAPDH

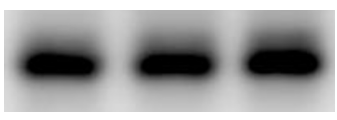

MDA-MB-231

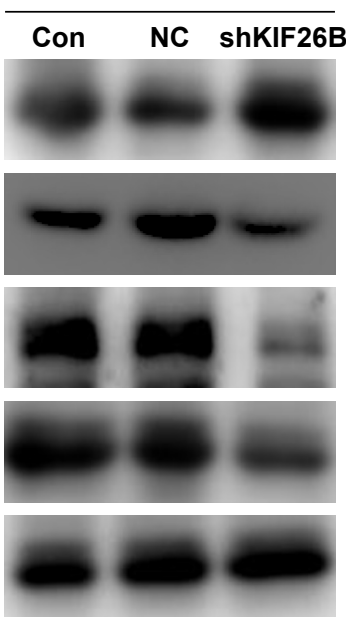

Figure 5 Knockdown of KIF26B inhibits tumor metastasis and invasion by modulating EMT-related genes.

Notes: (A) Transwell migration and Matrigel invasion assays using MCF-7 cells (left panel) and MDA-MB-23I cells (right panel) infected with KIF26B shRNA or NC. Scale bars, $100 \mu \mathrm{m}$ and representative images were taken under $\times 100$ magnification. (B) Migrated and invaded cells were counted in five random fields. Three independent experiments were performed, and the results are presented as the mean $\pm \mathrm{SD}$. ${ }^{*} p<0.05,{ }^{*} p<0.00 \mathrm{I}$. (C) Western blot analysis of the protein levels of EMT-related genes, including E-cadherin, N-cadherin, vimentin, and Snaill, in MCF-7 and MDA-MB-23I cells infected with KIF26B shRNA or NC. GAPDH served as an internal control.

Abbreviations: Con, control; EMT, epithelial-mesenchymal transition; KIF26B, kinesin family member 26B; NC, negative control.

Our findings indicate that KIF26B may drive EMT in breast cancer cells, resulting in metastasis.

Overexpression of KIF26B in breast cancer tissues has been reported. ${ }^{15}$ However, the mechanisms regulating KIF26B expression remain unknown. As Sall1 is a transcriptional activator of KIF26B, ${ }^{10}$ it would be interesting to investigate the relationship between KIF26B and Sall1 in breast cancer. Recently, Pu et $\mathrm{al}^{26}$ demonstrated that KIF26B is a direct target of miR-20a-5p and plays a key role in multidrug resistance in osteosarcoma. We believe that KIF26B may also contribute to multidrug resistance in breast cancer. In summary, KIF26B may be a promising target for breast cancer treatment. However, further studies are needed to fully understand the oncogenic role of KIF26B in breast cancer.

\section{Disclosure}

The authors report no conflicts of interest in this work.

\section{References}

1. Ferlay J, Soerjomataram I, Dikshit R, et al. Cancer incidence and mortality worldwide: sources, methods and major patterns in GLOBOCAN 2012. Int J Cancer. 2015;136:E359-E386.

2. Siegel R, Ma J, Zou Z, Jemal A. Cancer statistics, 2014. CA Cancer J Clin. 2014;64:9-29.

3. Fan L, Strasser-Weippl K, Li JJ, et al. Breast cancer in China. Lancet Oncol. 2014;15:e279-e289.

4. Parker JS, Mullins M, Cheang MC, et al. Supervised risk predictor of breast cancer based on intrinsic subtypes. J Clin Oncol. 2009;27:1160-1167.

5. Cuzick J, DeCensi A, Arun B, et al. Preventive therapy for breast cancer: a consensus statement. Lancet Oncol. 2011;12:496-503.

6. Shimelis H, Mesman RLS, Von Nicolai C, et al. BRCA2 Hypomorphic missense variants confer moderate risks of breast cancer. Cancer Res. 2017;77:2789-2799.

7. Weigelt B, Peterse JL, van 't Veer LJ. Breast cancer metastasis: markers and models. Nat Rev Cancer. 2005;5:591-602.

8. Miki H, Setou M, Kaneshiro K, Hirokawa N. All kinesin superfamily protein, KIF, genes in mouse and human. Proc Natl Acad Sci U S A. 2001;98:7004-7011.

9. Hirokawa N, Noda Y, Tanaka Y, Niwa S. Kinesin superfamily motor proteins and intracellular transport. Nat Rev Mol Cell Biol. 2009;10: 682-696. 
10. Uchiyama Y, Sakaguchi M, Terabayashi T, et al. Kif26b, a kinesin family gene, regulates adhesion of the embryonic kidney mesenchyme. Proc Natl Acad Sci U S A. 2010;107:9240-9245.

11. Heinrich J, Proepper C, Schmidt T, Linta L, Liebau S, Boeckers TM. The postsynaptic density protein Abelson interactor protein 1 interacts with the motor protein Kinesin family member 26B in hippocampal neurons. Neuroscience. 2012;221:86-95.

12. Gu J, Ajani JA, Hawk ET, et al. Genome-wide catalogue of chromosomal aberrations in barrett's esophagus and esophageal adenocarcinoma: a high-density single nucleotide polymorphism array analysis. Cancer Prev Res. 2010;3:1176-1186.

13. Wang J, Cui F, Wang X, et al. Elevated kinesin family member 26B is a prognostic biomarker and a potential therapeutic target for colorectal cancer. J Exp Clin Cancer Res. 2015;34:13.

14. Zhang H, Ma RR, Wang XJ, et al. KIF26B, a novel oncogene, promotes proliferation and metastasis by activating the VEGF pathway in gastric cancer. Oncogene. 2017;36:5609-5619.

15. Wang Q, Zhao ZB, Wang G, Hui Z, Wang MH, Pan JF, Zheng H. High expression of KIF26B in breast cancer associates with poor prognosis. PLoS One. 2013;8:e61640.

16. Xie Y, Wang B. Downregulation of TNFAIP2 suppresses proliferation and metastasis in esophageal squamous cell carcinoma through activation of the Wnt/ $\beta$-catenin signaling pathway. Oncol Rep. 2017;37: 2920-2928.

17. Wang J, Ma S, Ma R, et al. KIF2A silencing inhibits the proliferation and migration of breast cancer cells and correlates with unfavorable prognosis in breast cancer. BMC Cancer. 2014;14:461.
18. Corson TW, Gallie BL. KIF14 mRNA expression is a predictor of grade and outcome in breast cancer. Int J Cancer. 2006;119:1088-1094.

19. Wang C, Wang C, Wei Z, et al. Suppression of motor protein KIF3C expression inhibits tumor growth and metastasis in breast cancer by inhibiting TGF- $\beta$ signaling. Cancer Lett. 2015;368:105-114.

20. Bloom J, Cross FR. Multiple levels of cyclin specificity in cell-cycle control. Nat Rev Mol Cell Biol. 2007;8:149-160.

21. Nigg EA. Cyclin-dependent protein kinases: key regulators of the eukaryotic cell cycle. Bioessays. 1995;17:471-480.

22. Ezhevsky SA, Ho A, Becker-Hapak M, Davis PK, Dowdy SF. Differential regulation of retinoblastoma tumor suppressor protein by $\mathrm{G}(1)$ cyclin-dependent kinase complexes in vivo. Mol Cell Biol. 2001;21: 4773-4784.

23. Zheng L, Zheng J, Wu LJ, Zhao YY. Julibroside J8-induced HeLa cell apoptosis through caspase pathway. $J$ Asian Nat Prod Res. 2006;8: $457-465$.

24. Xia H, Ooi LL, Hui KM. MicroRNA-216a/217-induced epithelialmesenchymal transition targets PTEN and SMAD7 to promote drug resistance and recurrence of liver cancer. Hepatology. 2013;58: 629-641.

25. Lee JM, Dedhar S, Kalluri R, Thompson EW. The epithelial-mesenchymal transition: new insights in signaling, development, and disease. J Cell Biol. 2006;172:973-981.

26. Pu Y, Yi Q, Zhao F, Wang H, Cai W, Cai S. MiR-20a-5p represses multi-drug resistance in osteosarcoma by targeting the KIF26B gene. Cancer Cell Int. 2016;16:64.
OncoTargets and Therapy

\section{Publish your work in this journal}

OncoTargets and Therapy is an international, peer-reviewed, open access journal focusing on the pathological basis of all cancers, potential targets for therapy and treatment protocols employed to improve the management of cancer patients. The journal also focuses on the impact of management programs and new therapeutic agents and protocols on

\section{Dovepress}

patient perspectives such as quality of life, adherence and satisfaction. The manuscript management system is completely online and includes a very quick and fair peer-review system, which is all easy to use. Visit http://www.dovepress.com/testimonials.php to read real quotes from published authors. 